\title{
Limited-data x-ray CT for underwater pipeline inspection
}

Riis, N. A.B.; Frøsig, J.; Dong, Y.; Hansen, P. C.

\section{Published in:}

Inverse Problems

Link to article, DOI:

10.1088/1361-6420/aaa49c

Publication date:

2018

Document Version

Peer reviewed version

Link back to DTU Orbit

Citation (APA):

Riis, N. A. B., Frøsig, J., Dong, Y., \& Hansen, P. C. (2018). Limited-data x-ray CT for underwater pipeline inspection. Inverse Problems, 34(3), [034002]. https://doi.org/10.1088/1361-6420/aaa49c

\section{General rights}

Copyright and moral rights for the publications made accessible in the public portal are retained by the authors and/or other copyright owners and it is a condition of accessing publications that users recognise and abide by the legal requirements associated with these rights.

- Users may download and print one copy of any publication from the public portal for the purpose of private study or research.

- You may not further distribute the material or use it for any profit-making activity or commercial gain

- You may freely distribute the URL identifying the publication in the public portal

If you believe that this document breaches copyright please contact us providing details, and we will remove access to the work immediately and investigate your claim. 


\title{
Limited-Data X-Ray CT for Underwater Pipeline Inspection
}

\author{
N A B Riis ${ }^{1}$, J Frøsig ${ }^{2}$, Y Dong ${ }^{1}$ and P C Hansen ${ }^{1}$ \\ ${ }^{1}$ Department of Applied Mathematics and Computer Science, Richard Petersens \\ Plads, Building 324, DK-2800 Kgs. Lyngby, Denmark \\ 2 FORCE Technology, Park Alle 345, DK-2605 Brøndby, Denmark \\ E-mail: nabr@dtu.dk, jafs@force.dk, yido@dtu.dk and pcha@dtu.dk
}

\begin{abstract}
Tomographic reconstruction from limited data is an important problem that arises in many applications and can be handled in many ways. Here we consider inspection of underwater oil pipelines via fan-beam X-ray CT where, due to restrictions in the measurement device, the beam cannot illuminate the full area to be reconstructed. Moreover, it is desirable to use only a small number of projections to save measurement time. We use microlocal analysis to determine a favorable scanning geometry, and propose a reconstruction method based on compactly supported shearlets with a weighted sparsity penalty. Numerical simulations and results on real data demonstrate the usefulness of our approach and that we are able to locate defects in the pipe from a small number of projections.
\end{abstract}

AMS classification scheme numbers: $65 \mathrm{~K} 10,65 \mathrm{~F} 22$

8 December 2017

Keywords: Computed tomography, inverse problems, limited data, microlocal analysis, shearlet, sparsity, ADMM method.

Submitted to: Inverse Problems 

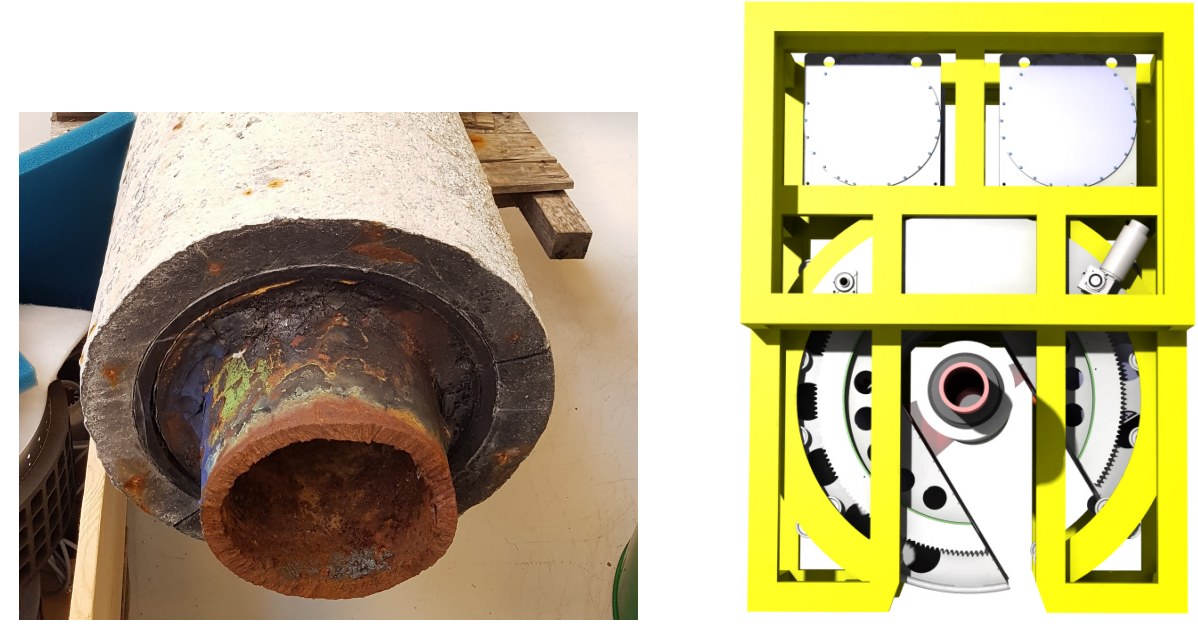

Figure 1. Left: a typical underwater oil pipe. Right: an outline of the planned X-ray inspection device. The pictures are kindly provided by FORCE Technology.

\section{Introduction}

Our work is motivated by the need to inspect underwater oil pipelines for defects and cracks in the steel pipe and the protective layers of rubber and concrete, as well as congestion or residue buildup in the central regions - see the picture in Figure 1. This is currently accomplished by a diver with an ultrasound device, but it is impractical because it is time consuming and because it is often necessary to remove part of the pipes outer layers. An alternative is to use a remotely controlled X-ray inspection device, outlined in Figure 1, that generates cross-sectional images of the pipe in real time using Computed Tomography (CT).

Since the pipe consists of materials with high absorption coefficients, it is necessary to use a powerful X-ray source with a narrow fan beam. Thus the beam cannot illuminate the whole pipe (in contrast to regular CT where the beam illuminates the entire object), which is the main challenge in this application. Therefore a central question is how to illuminate the pipe with the narrow fan beam, and we demonstrate how microlocal analysis provides important information to guide the decision.

The use of microlocal analysis in CT has a long history, and as far as we know it starts from $[1,2]$. More recently, insight from microlocal analysis has been used to provide algorithms that improve reconstructions for certain limited-data problems $[3,4,5]$. For limited-data problems where the limitation in reconstruction quality is intrinsic to the underlying tomographic setup, the chapter by Krishnan and Quinto in [6] provides a microlocal analysis framework that can quantify the extend of this limitation.

Another challenging issue is a desire to save measurement time by reducing the number of projections. The question is how many - or few - projections are needed in order to compute an acceptable cross-section image. If we use a small number of projections then we face a highly underdetermined reconstruction problem that calls 
for regularization. Enforcing sparsity in some representation of the CT image has been widely used for regularization in limited-data tomography. Promising reconstruction results have been obtained for specific limited-data problems that utilize curvelets [7] or Haar wavelets [8] with a sparsity penalty. Furthermore, the use of shearlets to represent reconstruction from limited data has also recently been proposed either in conjunction with total variation regularization $[9,10]$ or as a stand-alone sparsity constraint on the shearlet coefficients of the image [11] or the sinogram [12].

In this paper, we focus on the reconstruction problem of oil pipes. Based on the geometry of the pipe, we apply microlocal analysis to guide our decision on how to illuminate it, and propose a new off-center scanning geometry which still only illuminates approximately one half of the pipe, but is able to capture most cracks and defects in a full rotation. In order to resolve the cracks and defects in the pipe, we choose to represent the image by means of compactly supported shearlets, and propose a corresponding reconstruction method that enforces regularization via a sparsity penalty on the shearlet coefficients. Since compactly supported shearlets have been shown to be optimally sparse for representing discontinuities along curved edges in images [13], we are able to resolve most cracks and defects in the pipe even with few projections.

Our paper is organized as follows. In Section 2 we summarize the main results and conclusions from microlocal analysis applied to the specific pipeline inspection problem. In Section 3 we present the numerical method for computing a shearlet-based reconstruction with a weighted sparsity penalty. In particular, we discuss a proper choice of weights on the shearlet coefficients that is suited for the pipeline inspection

problem. In Section 4 we present numerical results from simulated and real measured underwater pipeline data. Finally, conclusions are drawn in Section 5.

\section{Off-Center Beam According to Microlocal Analysis}

Microlocal analysis (MLA) can be used to determine which singular features such as edges we can expect to recover in a range of continuous tomography problems. In this section, we first review the main definitions and theories in MLA relevant to the pipeline inspection problem, and then based on them propose a new off-center scanning geometry.

For a given angle $\varphi \in[0,2 \pi)$ we define the two unit vectors in $\mathbb{R}^{2}$ :

$$
\boldsymbol{\theta}(\varphi)=(\cos \varphi, \sin \varphi) \quad \text { and } \quad \boldsymbol{\theta}^{\perp}(\varphi)=\boldsymbol{\theta}(\varphi+\pi / 2) .
$$

Define the set of compactly supported distributions as $\mathcal{D}(X)$ with $X \subset \mathbb{R}^{2}$. For a distribution $f \in \mathcal{D}(X)$, its $2 D$ Radon transform $\mathcal{R}:[0,2 \pi) \times \mathbb{R} \rightarrow \mathbb{R}$ is defined by

$$
(\mathcal{R} f)(\varphi, s)=\int_{\mathbb{R}} f\left(s \boldsymbol{\theta}(\varphi)+t \boldsymbol{\theta}^{\perp}(\varphi)\right) \mathrm{d} t .
$$

The sinogram is the collection of measurement values modeled by the Radon transform as a function of $\varphi$ and $s$. In addition, we define the wavefront set of $f$ by

$$
\operatorname{WF}(f)=\left\{(\boldsymbol{\chi}, \boldsymbol{\xi}) \in X \times\left(\mathbb{R}^{2} \backslash\{\boldsymbol{0}\}\right): \boldsymbol{\xi} \in \Sigma_{\boldsymbol{\chi}}(f)\right\},
$$


where $\Sigma_{\boldsymbol{\chi}}(f)$ is the localized frequency set for $f$, cf. Definition 3.2 in [3]. Intuitively we can think of the wavefront set of $f$ in (3) as the set of singularities at position $\chi$ and with direction $\boldsymbol{\xi}$.

Now, we state the main result from MLA relevant for our reconstruction problem; see [6] for more details.

- If $\left(\chi_{0}, \boldsymbol{\xi}_{0}\right) \in \operatorname{WF}(f)$ then

$$
\left(\left(\varphi_{0}, \boldsymbol{\chi}_{0} \cdot \boldsymbol{\theta}\left(\varphi_{0}\right)\right),\left(-\alpha \boldsymbol{\chi}_{0} \cdot \boldsymbol{\theta}^{\perp}\left(\varphi_{0}\right), \alpha\right)\right) \in \operatorname{WF}(\mathcal{R} f),
$$

where the angle $\varphi_{0}$ and the scalar $\alpha \neq 0$ are chosen such that $\boldsymbol{\xi}_{0}=\alpha \boldsymbol{\theta}\left(\varphi_{0}\right)$. That is, a singularity in $f$ gives rise to a singularity in the sinogram if we measure along a line through the singularity, orthogonal to its direction.

- Let $\left(\varphi_{0}, s_{0}\right) \in[0,2 \pi] \times \mathbb{R}$ and $t_{0} \in \mathbb{R}$. If

$$
\left(\left(\varphi_{0}, s_{0}\right),\left(-\alpha t_{0}, \alpha\right)\right) \in \operatorname{WF}(\mathcal{R} f)
$$

for some $\alpha \neq 0$ then

$$
\left(\chi_{0}, \boldsymbol{\xi}_{0}\right) \in \mathrm{WF}(f)
$$

where

$$
\chi_{0}=s_{0} \boldsymbol{\theta}\left(\varphi_{0}\right)+t_{0} \boldsymbol{\theta}^{\perp}\left(\varphi_{0}\right), \quad \boldsymbol{\xi}_{0}=\alpha \boldsymbol{\theta}\left(\varphi_{0}\right) .
$$

That is, given a singularity in the sinogram there exists a singularity in $f$ that generated it.

In other words, a singularity $\left(\boldsymbol{\chi}_{0}, \boldsymbol{\xi}_{0}\right) \in \mathrm{WF}(f)$ - at position $\boldsymbol{\chi}_{0}$ and with direction $\boldsymbol{\xi}_{0}$ - is visible from $2 \mathrm{D}$ Radon data if and only if data from the line through $\boldsymbol{\chi}_{0}$ and perpendicular to $\boldsymbol{\xi}_{0}$ is measured.

Turning to the pipeline inspection problem, we first consider a scanning geometry where the center of the fan beam goes through the pipe's center for all projection angles. This is illustrated in the top left part of Figure 2, where the red lines visualize the Xrays and the red curve above the image is the measured data for that specific pipe and projection angle. The singularities of the pipe that are visible from this projection angle are shown by the red dots in the top middle part of Figure 2. The singularities that can be recovered from a full set of projection angles are shown by red in the top right part of the figure. Clearly, with this geometry we cannot hope to locate any singularities outside the central fully illuminated part of the pipe.

In order to inspect the complete pipe with the same fan beam, we propose an alternative scanning geometry with an off-center beam that illuminates approximately one half of the pipe, while keeping the center of rotation at the center of the pipe. This is illustrated in the bottom left part of Figure 2, where again the red lines visualize the $\mathrm{X}$-ray fan beam and the red curve above the figure is the measured data for that specific pipe and projection angle. The bottom middle and right parts show the singularities that are visible from this projection angle, and the singularities that can be recovered from a full set of projection angles. With this geometry and a full set of projection angles, all 


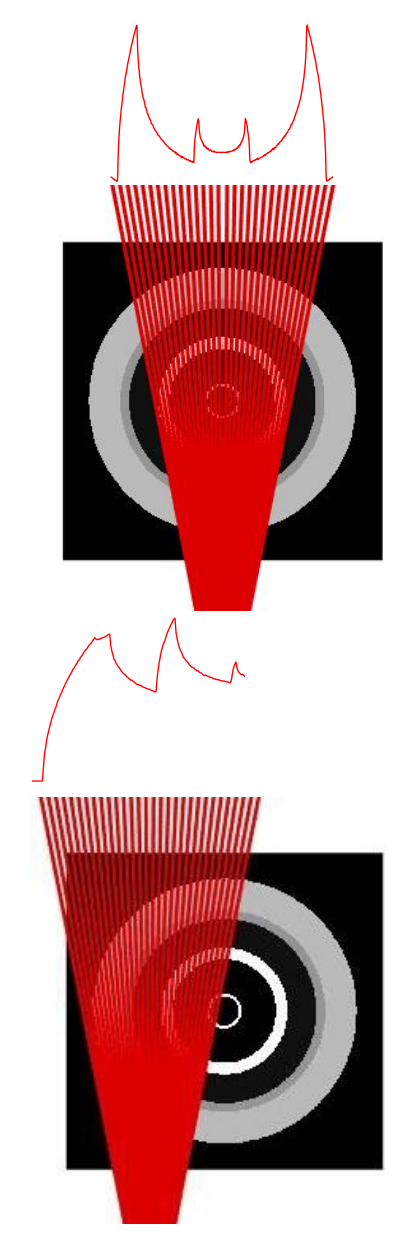

Measured data from one projection angle.
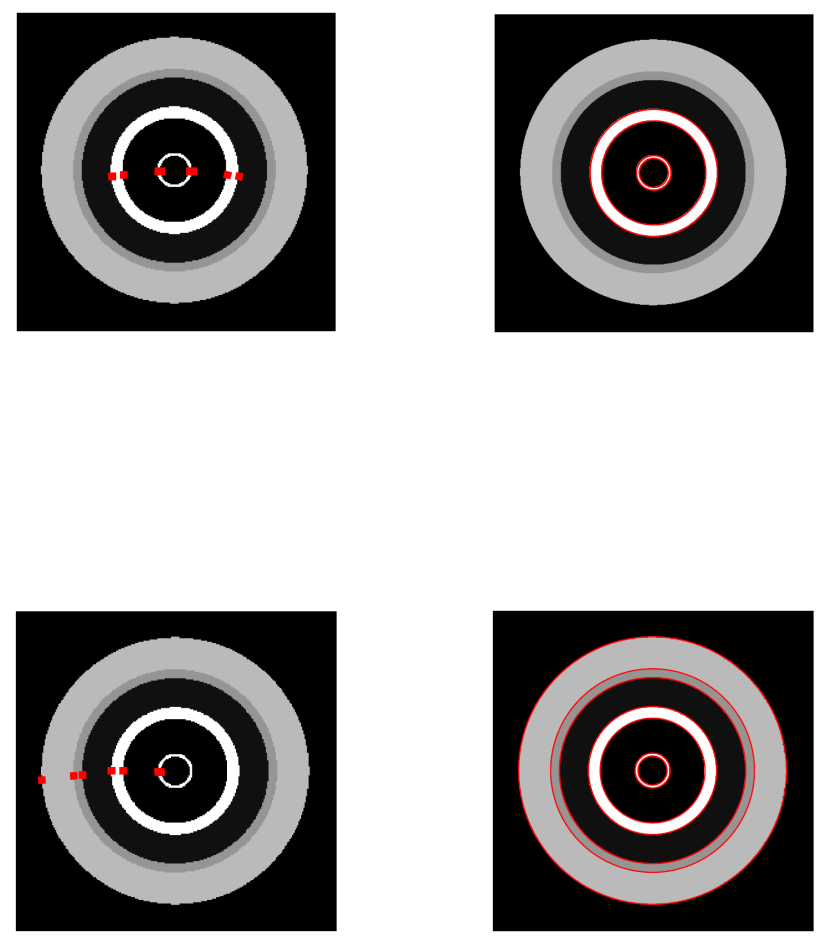

Singularities visible from Singularities visible from

one projection angle. all projection angles.

Figure 2. Illustration of the differences between the two scanning geometries. The red lines represent the actual fan beam in the scanner. Top: for a centered fan beam, we can only recover the singularities marked by red in the right plot. Bottom: for an off-center fan beam with the same width and center of rotation but a shift in source and detector position, we can - in principle - capture all the singularities in the pipe.

singularities of the pipe correspond to a singularity in the sinogram. Hence we are, in principle, able to capture all singularities of the pipe and can thus hope to reconstruct the pipe and most of the cracks and defects with the off-centered geometry. In Section 4, we will show that these predictions hold true through numerical experiments on simulated and real data with the centered and off-centered geometry.

\section{Reconstruction Method}

In X-ray tomography each measurement is modeled by the line integral along the corresponding X-ray over the unknown object. In the discretized setting, this process can be represented by a system matrix $\boldsymbol{A} \in \mathbb{R}^{m \times n}$, where $m$ is the total number of measurements and $n$ is the number of image pixels. This matrix can be computed with 

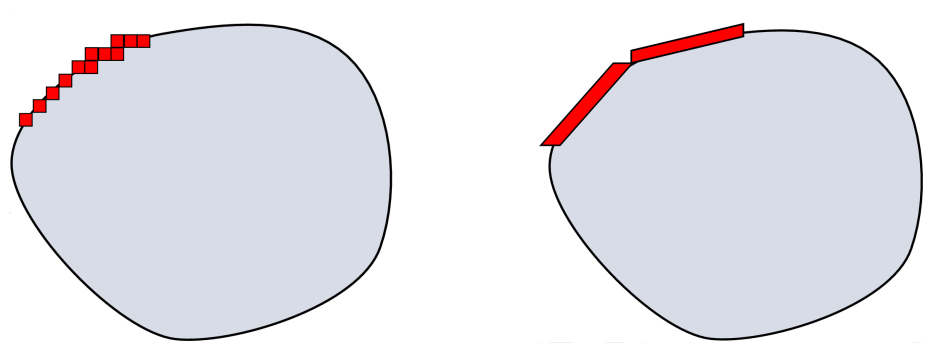

Figure 3. Representing a curved edge. Left: a pixel or Haar-wavelet basis (and similar non-directional bases) requires many elements. Right: a much sparser representation is possible with compactly supported shearlets.

the function fanbeamtomo from the AIR Tools package [14]. Thus, in principle, we arrive at the linear system $\boldsymbol{A} \boldsymbol{x} \approx \boldsymbol{b}$, where the vector $\boldsymbol{x} \in \mathbb{R}^{n}$ represents the object and $\boldsymbol{b} \in \mathbb{R}^{m}$ represents the measured noisy data.

Since we want to use a small number of projections but provide good resolution of the reconstruction, we end up with an underdetermined problem. Hence regularization is required in order to ensure a unique solution. Compactly supported shearlets have been shown to be optimally sparse for representing discontinuities along curved edges in images [13], and therefore we will utilize shearlets as the representation basis. Because of the effectiveness of shearlets, we expect that only a small number of basis functions are needed, i.e., the coefficients of the shearlet system for representing $\boldsymbol{x}$ are sparse. Thus, we arrive at the following discretized reconstruction problem:

$$
\min _{\boldsymbol{x} \geq \mathbf{0}} \frac{1}{2}\|\boldsymbol{A} \boldsymbol{x}-\boldsymbol{b}\|_{2}^{2}+\alpha\|\boldsymbol{W} \boldsymbol{\Phi} \boldsymbol{x}\|_{1},
$$

where $\alpha>0$ is the regularization parameter, $\boldsymbol{W}=\operatorname{diag}\left(w_{i}\right)$ is a $p \times p$ diagonal matrix with weights $w_{i} \geq 0, p$ is the number of frame elements in the corresponding shearlet system, and $\boldsymbol{\Phi} \in \mathbb{R}^{p \times n}$ denotes the associated shearlet analysis transform with $\boldsymbol{\Phi} \boldsymbol{x}$ as the shearlet coefficients (to be defined in Section 3.1). Here, we use the 2-norm in the first term of (8), i.e., the data-fitting term, because the noise in the data is assumed to be approximately additive Gaussian. The 1-norm in the second term is known to enforce sparsity on the shearlet coefficients [15]. The nonnegativity constraint in (8) reflects that the attenuation coefficients $\boldsymbol{x}$ cannot be negative.

\subsection{Review of Shearlets in $2 D$}

As already mentioned, shearlets provide a good representation basis for detecting the defects and cracks in the pipe. The motivations behind this are: 1) continuous shearlets have been shown to precisely capture the wavefront set of a distribution [16], which justifies the use of shearlets to detect singularities, and 2) compactly supported shearlets have been shown to be optimally sparse for representing images that are smooth except for singularities along curves [13]. Hence, shearlets are well suited for our problem because we can, in principle, represent all of the pipe and the defects with a small number 
of frame elements, because of the directional information encoded in the shearlets. An illustration of the intuition behind this is shown in Figure 3.

Now we briefly review the discrete shearlet systems in $2 \mathrm{D}$; readers who are unfamiliar with shearlets are referred to [17]. For $\phi, \psi, \tilde{\psi} \in L^{2}\left(\mathbb{R}^{2}\right)$ and $c=\left(c_{1}, c_{2}\right) \in \mathbb{R}_{+}^{2}$, the cone-adapted 2D discrete shearlet system $\mathrm{SH}(\phi, \psi, \tilde{\psi} ; c)$ is defined by

$$
\mathrm{SH}(\phi, \psi, \tilde{\psi} ; c)=\Phi\left(\phi ; c_{1}\right) \cup \Psi(\psi ; c) \cup \tilde{\Psi}(\tilde{\psi} ; c),
$$

where

$$
\begin{aligned}
& \Phi\left(\phi, c_{1}\right)=\left\{\phi_{m}=\phi\left(\cdot-c_{1} m\right): m \in \mathbb{Z}^{2}\right\}, \\
& \Psi(\psi ; c)=\left\{\psi_{j, k, m}=2^{\frac{3}{4} j} \psi\left(S_{k} A_{2^{j}} \cdot-M_{c} m\right): j \geq 0,|k| \leq\left\lceil 2^{j / 2}\right\rceil, m \in \mathbb{Z}^{2}\right\}, \\
& \tilde{\Psi}(\tilde{\psi} ; c)=\left\{\tilde{\psi}_{j, k, m}=2^{\frac{3}{4} j} \tilde{\psi}\left(\tilde{S}_{k} \tilde{A}_{2^{j}} \cdot-\tilde{M}_{c} m\right): j \geq 0,|k| \leq\left\lceil 2^{j / 2}\right\rceil, m \in \mathbb{Z}^{2}\right\},
\end{aligned}
$$

with

$$
A_{2^{j}}=\left[\begin{array}{cc}
2^{j} & 0 \\
0 & 2^{j / 2}
\end{array}\right], \quad S_{k}=\left[\begin{array}{cc}
1 & k \\
0 & 1
\end{array}\right], \quad M_{c}=\left[\begin{array}{cc}
c_{1} & 0 \\
0 & c_{2}
\end{array}\right]
$$

and

$$
\tilde{A_{2^{j}}}=\left[\begin{array}{cc}
2^{j / 2} & 0 \\
0 & 2^{j}
\end{array}\right], \quad \tilde{S}_{k}=\left[\begin{array}{cc}
1 & 0 \\
k & 1
\end{array}\right], \quad \tilde{M}_{c}=\left[\begin{array}{cc}
c_{2} & 0 \\
0 & c_{1}
\end{array}\right] .
$$

Here $\phi$ refers to the shearlet scaling function, while $\psi$ and $\tilde{\psi}$ are called shearlets. The parameters $j, k$ and $m$ represent the scaling, shearing and translation, respectively. The construction of a numerically stable shearlet system (consisting of compactly supported shearlets and associated analysis and synthesis transforms) depends on the choice of generating functions $\phi, \psi, \tilde{\psi}$ and $c$. In this paper we use the construction from the MATLAB library ShearLab [18]. Note that this shearlet system is not a tight frame, but is constructed in such a way that the analysis and synthesis operations are numerically stable in the sense of closeness to tightness.

By using the shearlets, we decompose the reconstruction $\boldsymbol{x}$ in (8) into its corresponding shearlet coefficients $\boldsymbol{c}=\boldsymbol{\Phi} \boldsymbol{x}$ with $\boldsymbol{c} \in \mathbb{R}^{p}$. Additionally, the reconstruction can be retrieved from the coefficients by applying $\boldsymbol{x}=\boldsymbol{\Phi}^{T} \boldsymbol{c}$, where the matrix $\boldsymbol{\Phi}^{T} \in \mathbb{R}^{n \times p}$ represents the associated synthesis transform. Note that if the shearlet system forms a tight frame then $\boldsymbol{x}=\boldsymbol{\Phi}^{T} \boldsymbol{\Phi} \boldsymbol{x}$, however for the compactly supported shearlets from [18] we only have $\boldsymbol{x} \approx \boldsymbol{\Phi}^{T} \boldsymbol{\Phi} \boldsymbol{x}$.

\subsection{ADMM Algorithm}

In order to solve the optimization problem in (8), we use the auxiliary variable $\boldsymbol{c}=\boldsymbol{\Phi} \boldsymbol{x}$ and solve the following constrained problem instead:

$$
\begin{array}{ll}
\min _{\boldsymbol{x}, \boldsymbol{c}} & \frac{1}{2}\|\boldsymbol{A} \boldsymbol{x}-\boldsymbol{b}\|_{2}^{2}+\alpha\|\boldsymbol{W} \boldsymbol{c}\|_{1} \\
\text { subject to } & \boldsymbol{\Phi} \boldsymbol{x}-\boldsymbol{c}=\mathbf{0} \quad \text { and } \quad \boldsymbol{x} \geq \mathbf{0} .
\end{array}
$$


This makes the optimization problem suited for applying the alternating direction method of multipliers (ADMM) [19].

Let $\boldsymbol{y} \in \mathbb{R}^{p}$ be the Lagrange multiplier for the constraint $\boldsymbol{\Phi} \boldsymbol{x}-\boldsymbol{c}=\mathbf{0}$. Then we have the corresponding augmented Lagrangian:

$$
\min _{\boldsymbol{x} \geq 0, \boldsymbol{c}, \boldsymbol{y}} \frac{1}{2}\|\boldsymbol{A} \boldsymbol{x}-\boldsymbol{b}\|_{2}^{2}+\alpha\|\boldsymbol{W} \boldsymbol{c}\|_{1}+\boldsymbol{y}^{T}(\boldsymbol{\Phi} \boldsymbol{x}-\boldsymbol{c})+\frac{\rho}{2}\left\|\boldsymbol{\Phi} \boldsymbol{x}-\boldsymbol{c}^{k}\right\|_{2}^{2},
$$

where $\rho>0$ is a penalty parameter. The scaled-form ADMM iterations, with the scaled Lagrange multiplier $\boldsymbol{u}=\rho^{-1} \boldsymbol{y}$, are then given by

$$
\begin{aligned}
\mathbf{x}^{k+1}:=\min _{\boldsymbol{x} \geq \mathbf{0}} \quad \frac{1}{2}\|\boldsymbol{A} \boldsymbol{x}-\boldsymbol{b}\|_{2}^{2}+\frac{\rho}{2}\left\|\boldsymbol{\Phi} \boldsymbol{x}-\boldsymbol{c}^{k}+\boldsymbol{u}^{k}\right\|_{2}^{2} \\
\mathbf{c}^{k+1}:=\min _{\mathbf{c}} \quad \alpha\|\boldsymbol{W} \boldsymbol{c}\|_{1}+\frac{\rho}{2}\left\|\boldsymbol{\Phi} \boldsymbol{x}^{k+1}-\boldsymbol{c}+\boldsymbol{u}^{k}\right\|_{2}^{2} \\
\boldsymbol{u}^{k+1}:=\boldsymbol{u}^{k}+\boldsymbol{\Phi} \boldsymbol{x}^{k+1}-\boldsymbol{c}^{k+1} .
\end{aligned}
$$

In each iteration, the computation is dominated by solving the two minimization subproblems in (17) and (18). Since the $\boldsymbol{x}$-subproblem in (17) is a least squares problem, we can approximate it efficiently by a CGLS method [20] and a non-negativity projection. In addition, the $\boldsymbol{c}$-subproblem in (18) has a closed-form solution by using element-wise soft thresholding:

$$
c_{i}^{k+1}:=\mathcal{S}_{\alpha w_{i} / \rho}\left((\boldsymbol{\Phi} \boldsymbol{x})_{i}+\boldsymbol{u}_{i}\right)
$$

with the soft-threshold operator $\mathcal{S}_{\kappa}(x)=\operatorname{sgn}(x) \max (|x|-\kappa, 0)$.

Because (8) is convex and its objective function is proper and closed, convergence of the ADMM algorithm is guarantied [19].

\subsection{The Choice of Weights $\boldsymbol{W}$}

The weights in the matrix $\boldsymbol{W}$ are chosen as a product of two different types of weights, i.e., $w_{i}=w_{i}^{\mathrm{s}} \cdot w_{i}^{\mathrm{r}}$ where $w_{i}^{\mathrm{s}}$ are called scale weights and $w_{i}^{\mathrm{r}}$ are ray-density weights.

The scale weights $w_{i}^{\mathrm{s}}$ depend solely on the scale, or level, of the corresponding shearlet. The key idea is that the smaller the support of the shearlet, the larger the weight. Specifically, for a given scale parameter $j$ and scale $2^{j}$, we set the scale weight:

$$
w_{j}^{\mathrm{s}}=2^{-j},
$$

effectively modifying the penalty of the shearlet coefficients by a factor of 1 at scale $2^{0}$,

$\frac{1}{2}$ at scale $2^{1}, \frac{1}{4}$ at scale $2^{2}, \frac{1}{8}$ at scale $2^{3}$, and so on. This idea for the scale weights is adapted from [7] and is meant to reflect the support of the shearlets.

The ray-density weights $w_{i}^{\mathrm{r}}$ reflect the X-ray "density" associated with the corresponding shearlet. This is done by calculating the relative density of the X-rays in each pixel and then integrating this density with the support of the shearlet, which leads to the definition

$$
w_{i}^{\mathrm{r}} \sim \mathcal{F}\left(\left\|\boldsymbol{D} \boldsymbol{r}_{i}\right\|_{2} /\left\|\boldsymbol{r}_{i}\right\|_{2}\right), \quad \boldsymbol{D}=\operatorname{diag}\left(\frac{\boldsymbol{s}-\min (\boldsymbol{s})}{\max (\boldsymbol{s}-\min (\boldsymbol{s}))}\right)
$$


where $\boldsymbol{s} \in \mathbb{R}^{p}$ with $s_{j}=\|A(:, j)\|_{1}$ and $\boldsymbol{r}_{i}^{T}$ is the $i$ th row of $\boldsymbol{\Phi}$, i.e., the vector representation of the i'th shearlet. The function $\mathcal{F}:[0,1] \rightarrow\left[1, w_{\max }\right]$ is given by $\mathcal{F}(t)=t+(1-t) w_{\max }$, where $w_{\max } \geq 0$ is a user-specified parameter. The idea is that shearlets with support in high ray-density areas are penalized less than ones that have support in lower density areas of the image. This effectively enforces stronger sparsity in areas with low ray density relative to the rest of the image. For the centered geometry this weighting scheme is similar to the one proposed in [8].

\section{Numerical Results}

This section provides the key numerical results associated with our analysis and experiments for the pipeline problem. For clarity we have divided the results into two objectives:

(i) Compare reconstruction quality for centered and off-centered geometries. Specifically, determine how switching to off-center geometry affects the quality of the reconstruction both in the center and the outer layers of the pipe.

(ii) Determine the effect that limiting the number of projections has on the reconstruction quality and find the smallest number of projections possible with an acceptable image quality.

In our method there are several parameters that need be chosen, including the regularization parameter $\alpha$, shearlet system $\mathrm{SH}(\phi, \psi, \tilde{\psi} ; c)$ and the parameter $w_{\max }$ in (22). The shearlet system is in all tests chosen to have 5 scales, i.e., $j=0,1 \ldots, 4$ with the generating shearlet being sheared 2 times in each direction for each cone in the first 3 scales (10 shearlets per scale) and 8 times in each direction for each cone in the last 2 scales (34 shearlets per scale). This is implemented by specifying the shearLevels array $[1,1,1,3,3]$ to the shearlet system generator in SHEARLAB [18]. The weight scheme is based on the scale and ray-density weights with $w_{\max }=5$, and we also give a comparison with the weight scheme to other choices of $w_{\max }$ and no scale weights in Section 4.4. In addition, unless otherwise noted, the regularization parameter $\alpha$ is chosen with respect to the best visual quality.

\subsection{Tomographic Setup}

Our goal is to get good reconstructions from real data, so we require our synthetic experiments to be as close to the real experiment as possible. Hence, we generate a phantom that resembles the actual oil pipe using absorption coefficients for the known pipe materials from the NIST database [21]. To avoid doing inverse crime we generate the data from a phantom on a high-resolution grid, and then we add Gaussian white noise to the data. The parameters defining the geometry and the system matrix $\boldsymbol{A}$ are listed in Table 1. The number of projection angles varies from 360 to 45, always distributed evenly between $0^{\circ}$ and $360^{\circ}$. The simulated data for 360 projections is compared to the actual measurements in Figure 4 for both scanning geometries. Data with fewer 
Table 1. Parameters defining the scanning geometry and the system matrix $\boldsymbol{A}$. The off-center shift is the distance the source and detector are shifted sideways in order to get the off-centered scanning geometry illustrated in the bottom half of Figure 2. The off-center shift is 0 for the centered scanning geometry.

\begin{tabular}{ll}
\hline Parameter & Value \\
\hline Reconstruction domain size & $55 \mathrm{~cm} \times 55 \mathrm{~cm}$ \\
Source to center distance & $59 \mathrm{~cm}$ \\
Source to detector distance & $100 \mathrm{~cm}$ \\
Detector length & $41.1 \mathrm{~cm}$ \\
Off-center shift & $13 \mathrm{~cm}$ \\
\hline Image pixels & $512 \times 512$ \\
Detector pixels & 512 \\
Number of projection angles & $360,180,90,45$ \\
\hline
\end{tabular}

projections are extracted from these data sets by removing every second projection to obtain 180 projections, and then removing every second to reach 90 projections, and so on. The synthetic phantom is shown in the top of Figure 5.

\subsection{Comparison of Centered and Off-Centered Scanning Geometries}

In Section 2 we hypothesized that the off-centered geometry would be able to capture all singularities of the pipe, while the centered geometry would only capture singularities inside the central fully illuminated part of the pipe. This is reflected in the synthetic and real sinograms shown in Figure 4, where the data from the off-centered geometry show new material layers that are not visible in the centered version of the data.

In addition to two different scanning geometries, we also compare our method with Kaczmarz's algorithm [22] with a non-negativity constraint, which does not involve any other prior information on the objects. The results from 180 projection angles are shown in Figure 5 with the phantom in the top of the figure. Notice that in the reconstructions using the data from the centered geometry, the outer layers of the pipe are invisible and one of the rings in the central region is brighter compared to the phantom. This would lead one to believe that the material has a higher absorption coefficient than it actually has, and that no absorption is happening outside the central region. This is because there is no way to determine the location of the material outside this central region when the singularities, or boundaries, of the material are not captured in the measurements. In contrast the off-centered geometry does capture the singularities outside the central region and hence reconstructions show all material layers of the pipe, while also giving absorption coefficients that are more in line with the phantom. Moreover, our shearletbased method (bottom row) visibly improves reconstruction compared to the Kaczmarz's algorithm (middle row). Essentially the use of shearlets reduces line artifacts from sparse angles, which are very clear in the reconstructions from Kaczmarz's algorithm, while it maintains the actual singularities from the phantom. Finally, since no X-rays hit the 

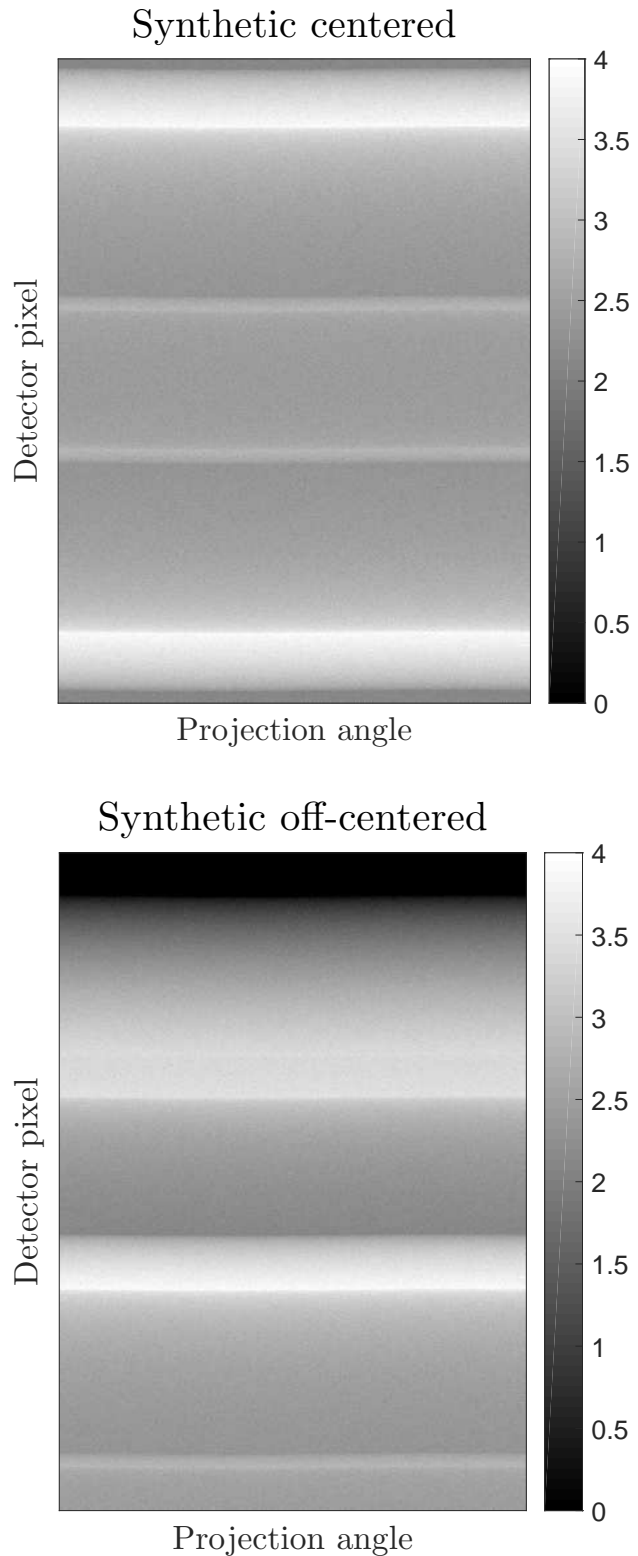
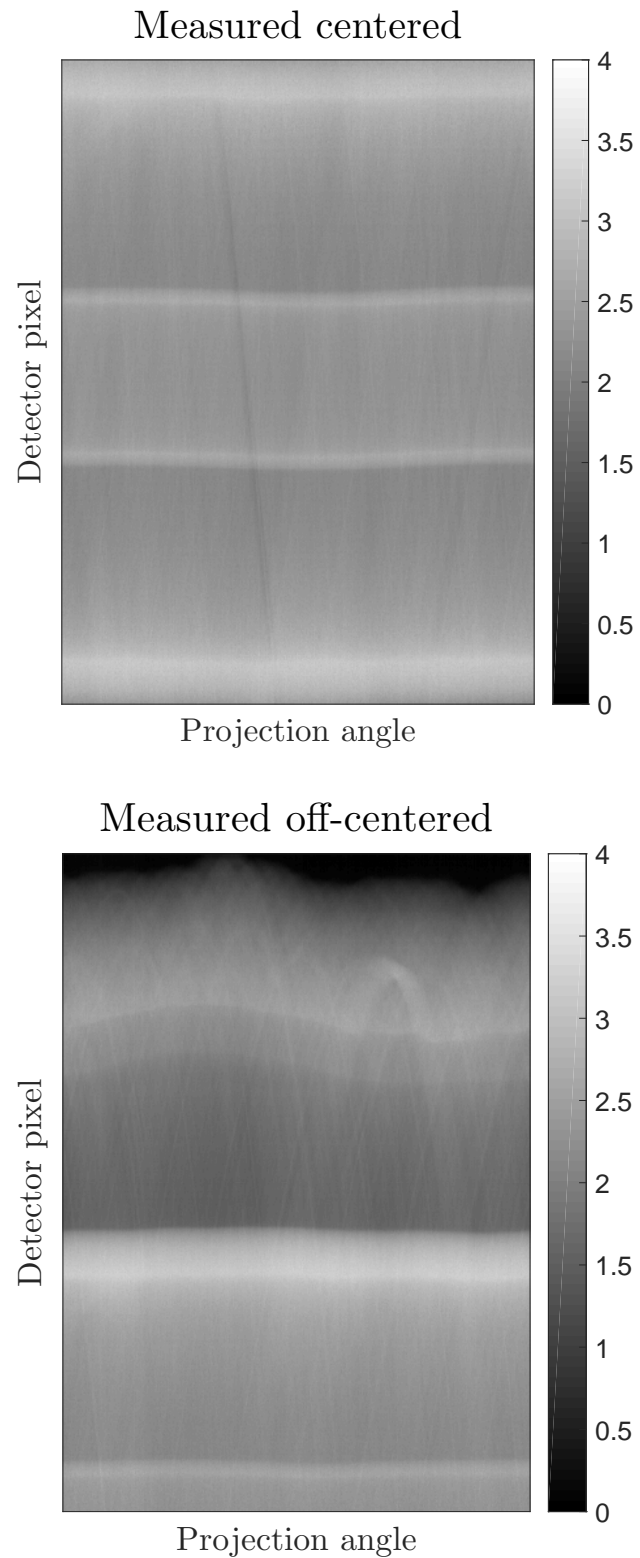

Figure 4. Synthetic (left) and measured (right) sinograms for centered (top) and off-centered (bottom) geometries with 360 projection angles.

very center of the pipe with the off-centered geometry, a small circular artifact show in the reconstruction from the Kaczmarz's algorithm near the center. This artifact is not present in our shearlet reconstruction.

Repeating the experiment with the real measured data from Figure 4 reduced to 180 projection angles, we obtain the reconstructions shown in Figure 6. The top image is the "ground truth" generated from a shearlet reconstruction from the off-centered data with 360 projection angles. The outer layers of the pipe are still invisible in the results from the centered geometry. Moreover, with 180 projection angles it is clear that our method gives improved reconstructions with the off-centered geometry. It maintains many small details in the outer layer of the pipe, and avoids the limited-data artifacts. The question 
remains, however, how few projections we can use while maintaining reasonable image quality.

\subsection{Limiting the Number of Projection Angles}

To study the effect of limiting the number of projections even further, we compare the reconstruction quality from the measured data generated from the off-centered geometry, reduced to 180, 90 and 45 projection angles. Due to the small number of projection angles, it becomes important to incorporate prior information on the objects in order to obtain good reconstruction. In this section, we compare our method with Kaczmarz's algorithm [22] and the L2-TV reconstruction method, which solves the variational model with the 2-norm data-fit term as in (15) and total variation (TV) regularization introduced in [23] by the Chambolle-Pock algorithm in [24]. All methods are solved under a non-negativity constraint. Kaczmarz's algorithm does not incorporate any other prior information on the objects, however, the L2-TV method is based on the assumption that the objects are piecewise constant. Figure 7 shows the zoomed parts from the reconstructions by using Kaczmarz's algorithm, the L2-TV method, and our shearlet method. We see that our method provides a reduction of the artifacts due to sparse projection angles compared to Kaczmarz's algorithm and also maintains more details of the pipe compared to the L2-TV method, e.g. the bright bar in the middle. In the results from the L2-TV method we can clearly see the typical staircasing artifacts. With a smaller regularization parameter the L2-TV method is able to preserve more details, but at the same time starts showing line artifacts similar to those in the Kaczmarz reconstruction, so we did not include those L2-TV results. When the number of projection angles is reduced to 45, some visible artifacts also appear in the results by our method. We therefore conclude that 90 projection angles are sufficient to detect reasonably large defects on the pipe when using the off-centered scanning geometry and our shearlet method.

\subsection{Effect of Parameter Selections}

In this section we study the effect on the quality of the reconstructions when varying the regularization parameter $\alpha$ and the weights $\boldsymbol{W}$.

Figure 8 shows the results by using our method with the regularization parameter varying from 0.001 to 0.022 . In our model (8), $\alpha$ controls the trade-off between a good fit to the data and the sparsity in shearlet representation. Hence, it is clear that if $\alpha$ is too small then more artifacts appear in the results, while if $\alpha$ is too large the reconstruction is too sparsely represented and many details are lost.

Figure 9 shows the reconstructions with different choices of the weights. We have tested our method with no weights at all (i.e., $\boldsymbol{W}$ is the identity), only scale weights (i.e., $w_{\max }=1$ ), and the weight scheme introduced in Section 3.3 with $w_{\max }=5$. From Figure 9 we can see that the scale weight plays an important role and it improves the reconstruction quality. Compared with the results from different values of $w_{\max }$, we see 


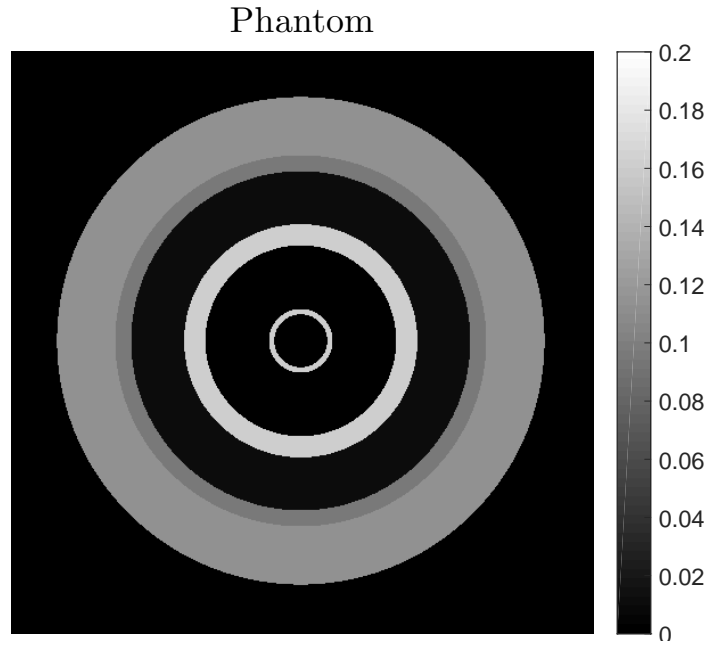

Kaczmarz, 3 iterations

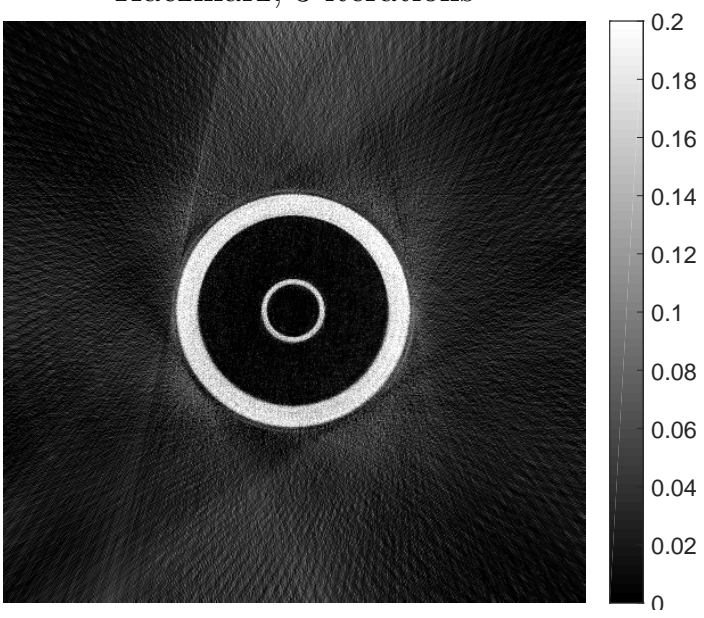

Shearlet, $\alpha=0.022$
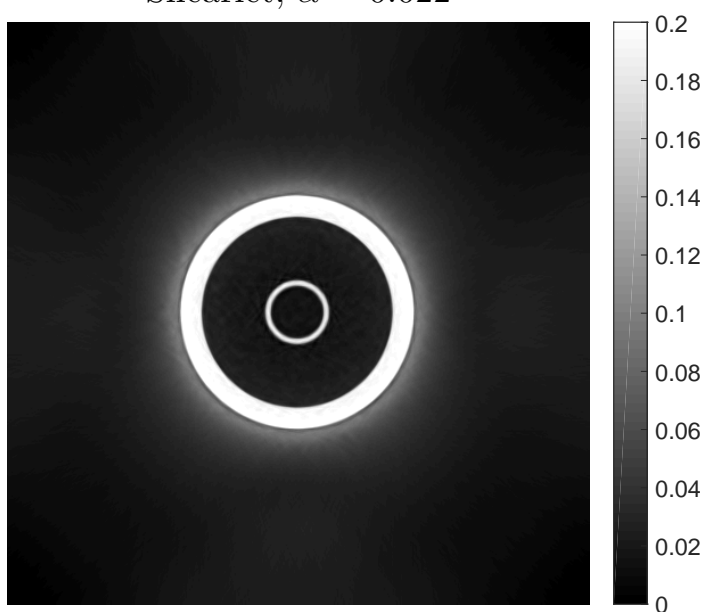

Kaczmarz, 3 iterations

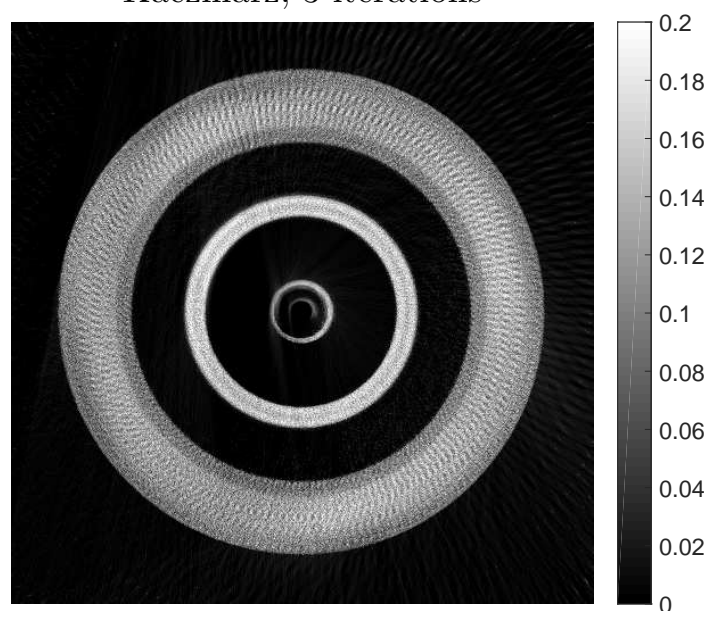

Shearlet, $\alpha=0.022$

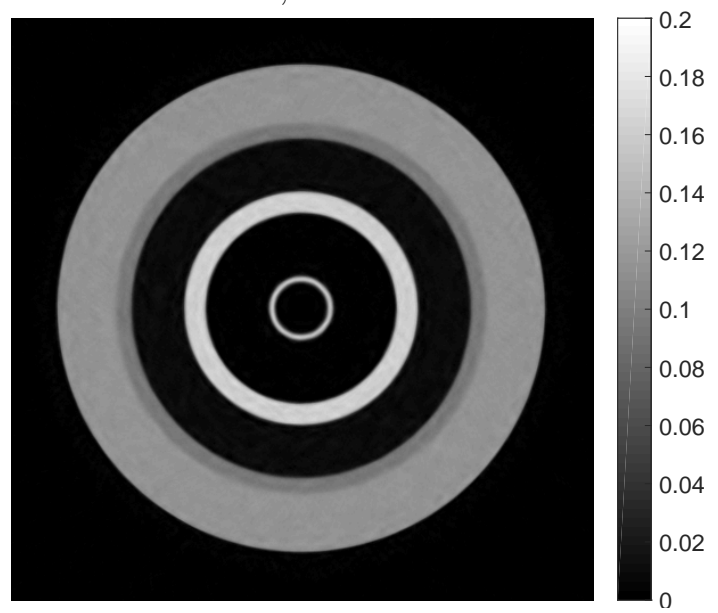

Figure 5. Reconstructions from synthetic data with $2 \%$ noise and 180 projection angles. Top: phantom. Middle: reconstructions from Kaczmarz's algorithm with centered (left) and off-centered (right) scanning geometries. Bottom: reconstructions from our method under the same geometries as above. 


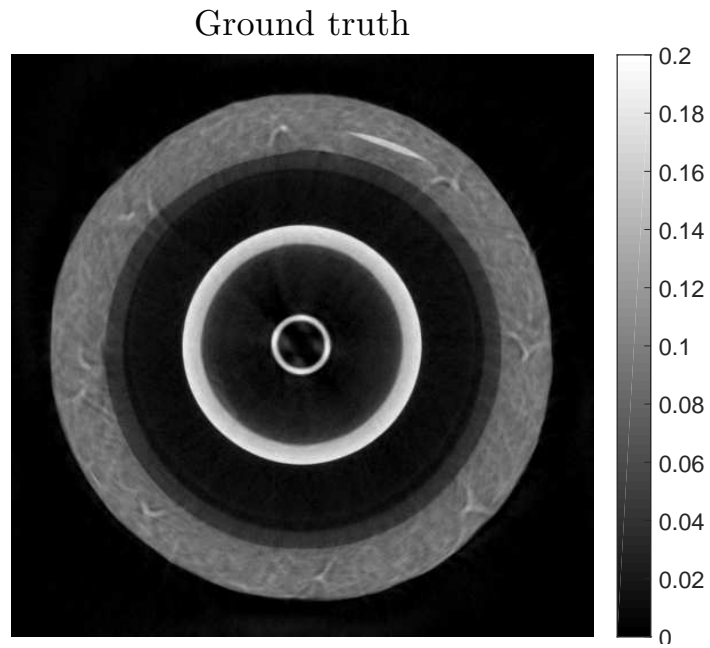

Kaczmarz, 5 iterations

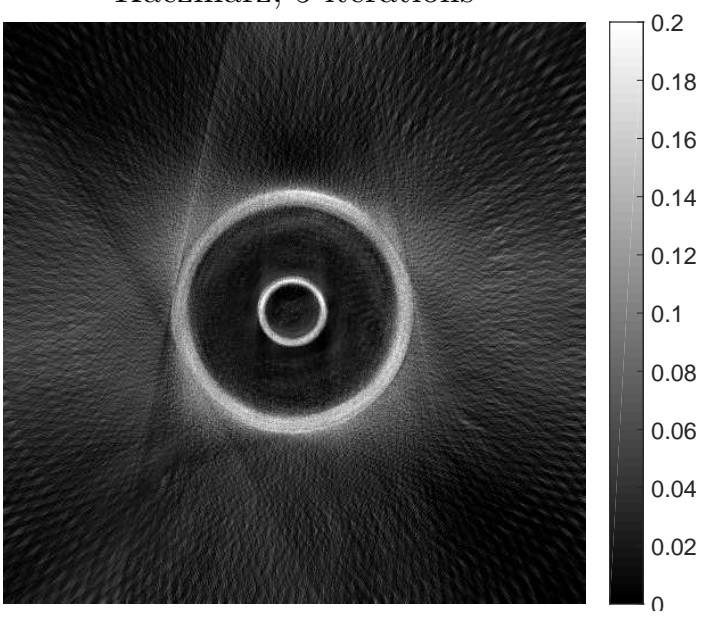

Shearlet, $\alpha=0.010$

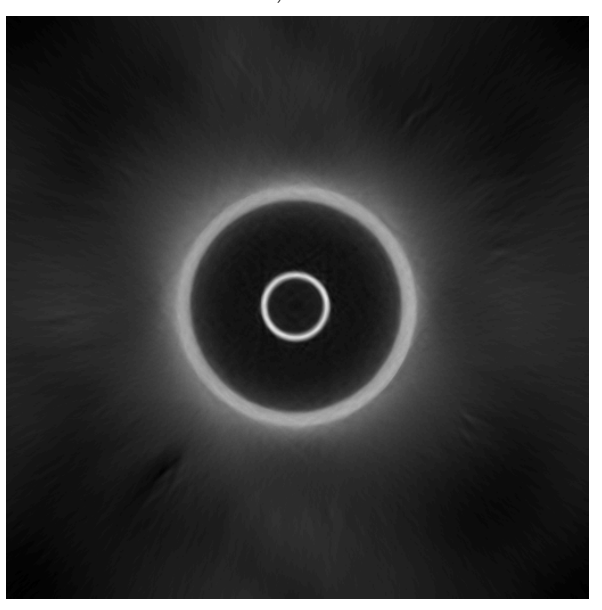

Kaczmarz, 5 iterations

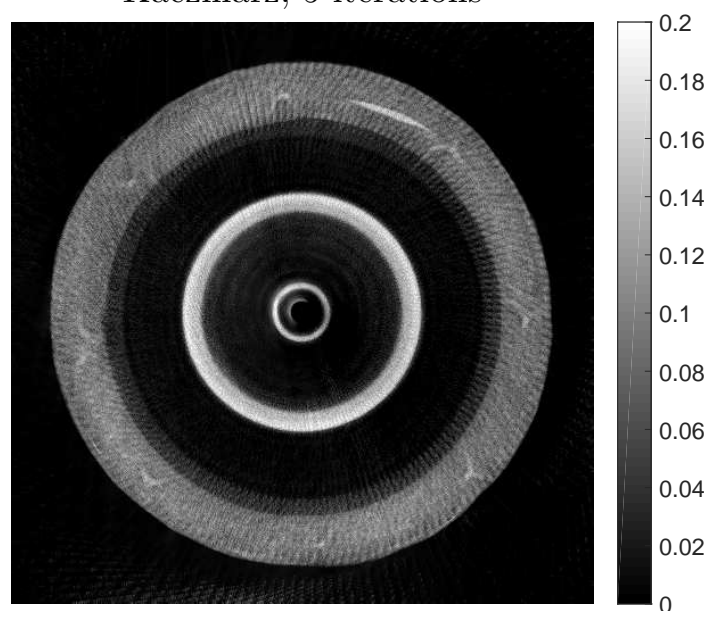

Shearlet, $\alpha=0.005$

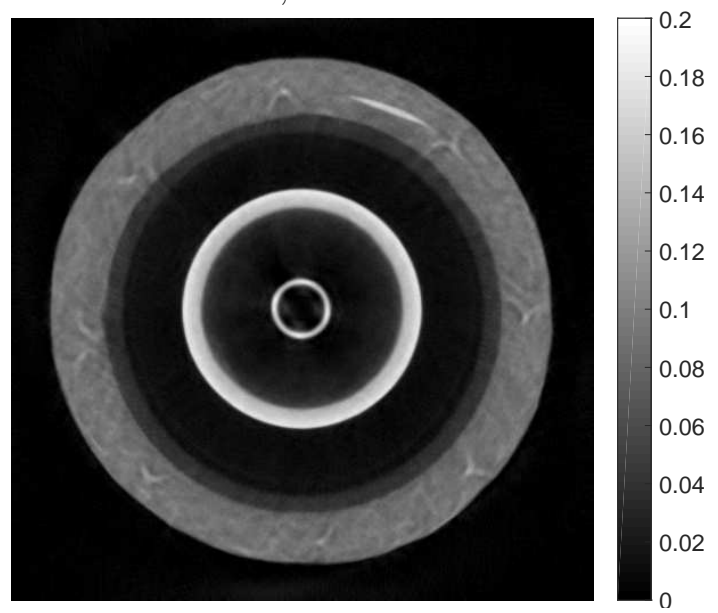

Figure 6. Reconstructions from real measured data with 180 projection angles. Top: "ground truth" reconstructed from off-center scanning geometry data with 360 projection angles. Middle: reconstructions from Kaczmarz's algorithm with centered (left) and off-centered (right) scanning geometries. Bottom: reconstructions from our method under the same geometries as above. 


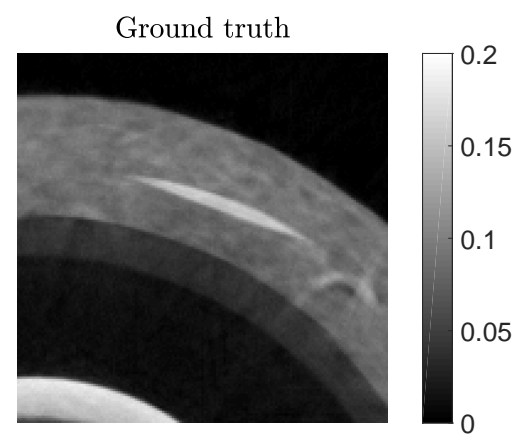

180: Kaczmarz, 5 iterations
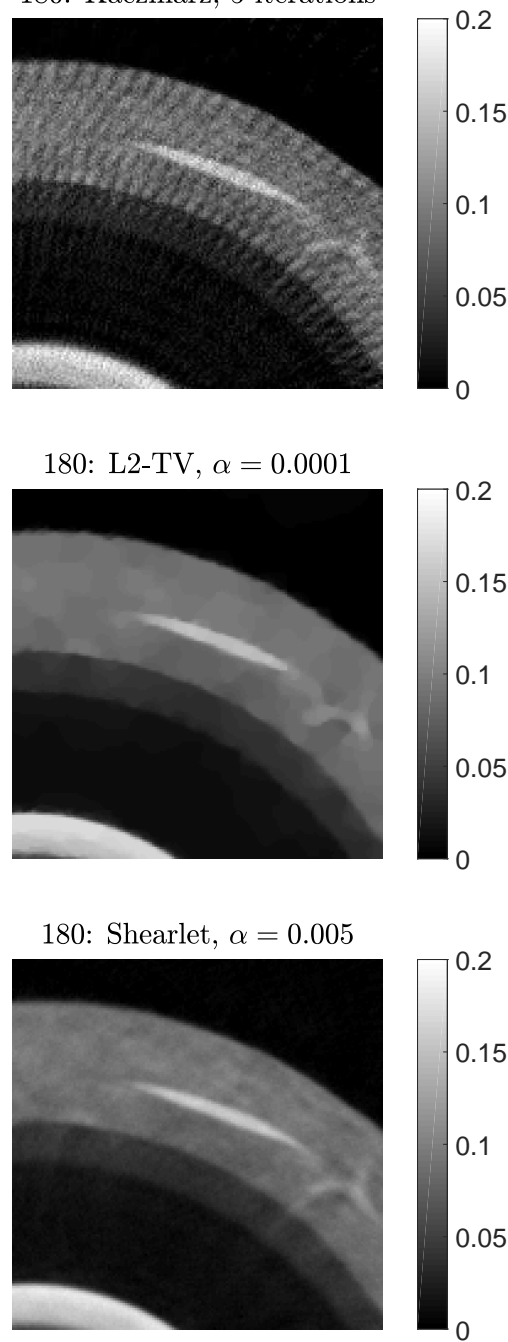

90: Kaczmarz, 6 iterations

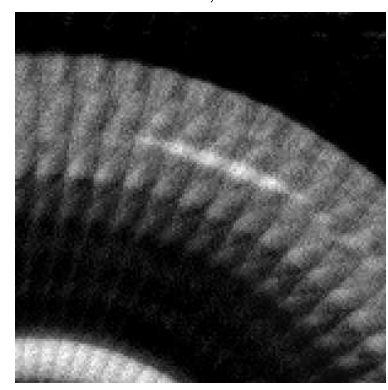

90: L2-TV, $\alpha=0.0001$

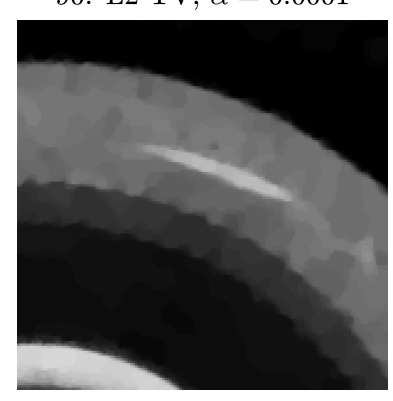

90: Shearlet, $\alpha=0.005$

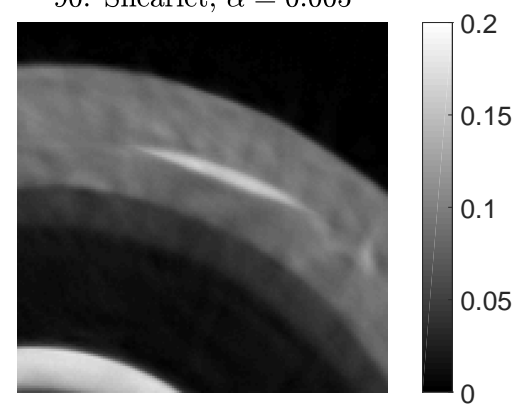

45: Kaczmarz, 8 iterations

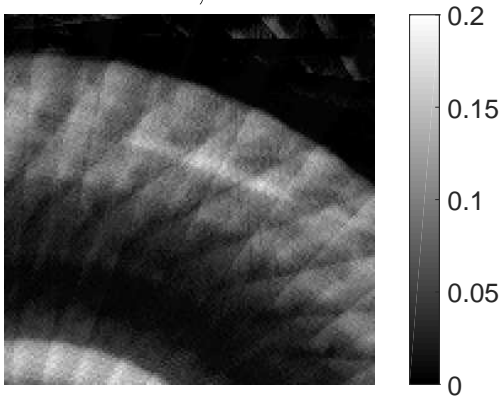

45: L2-TV, $\alpha=0.0005$

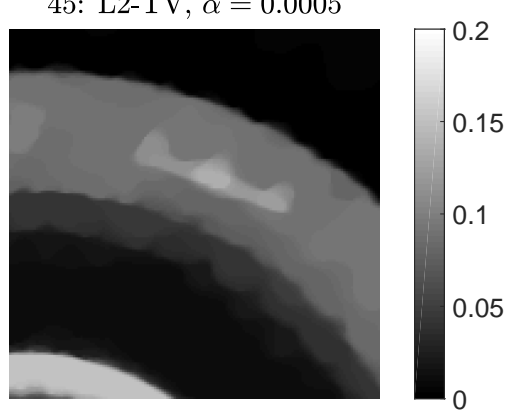

45: Shearlet, $\alpha=0.010$

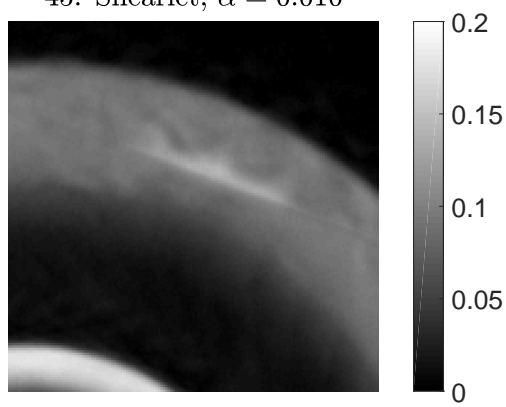

Figure 7. Zoomed parts of the reconstructions with sparse angles, using using Kaczmarz's algorithm, the L2-TV method, and our shearlet method.

The ground truth is shown at the top. The number of projection angles are 180, 90 and 45 from left to right, respectively. 

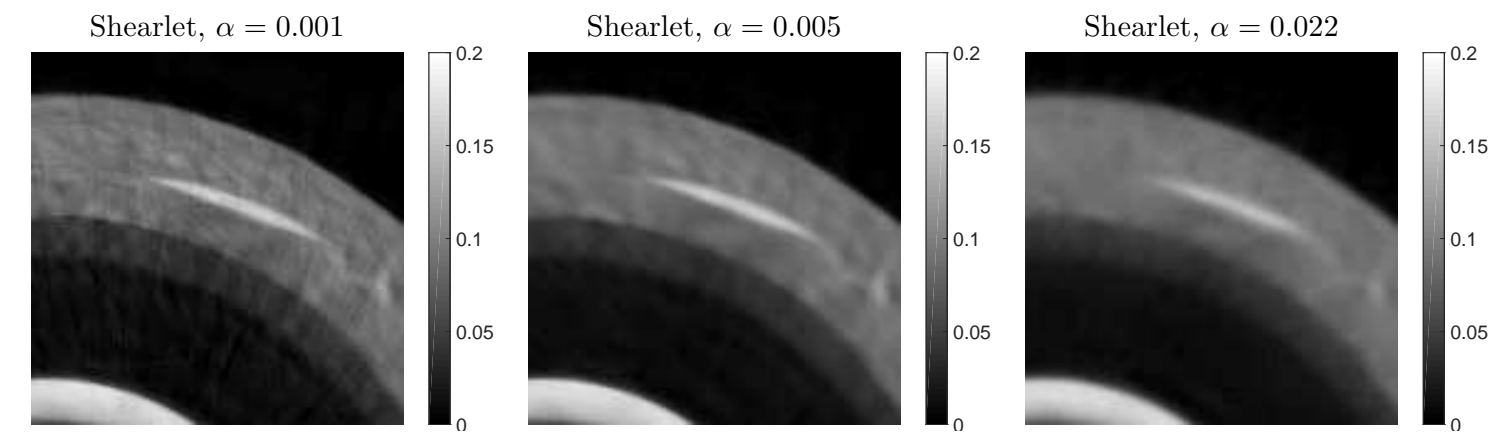

Figure 8. The effect of varying the regularization parameter $\alpha$ in our model (8) with 90 projection angles. From left to right $\alpha=0.001,0.005,0.022$, respectively.
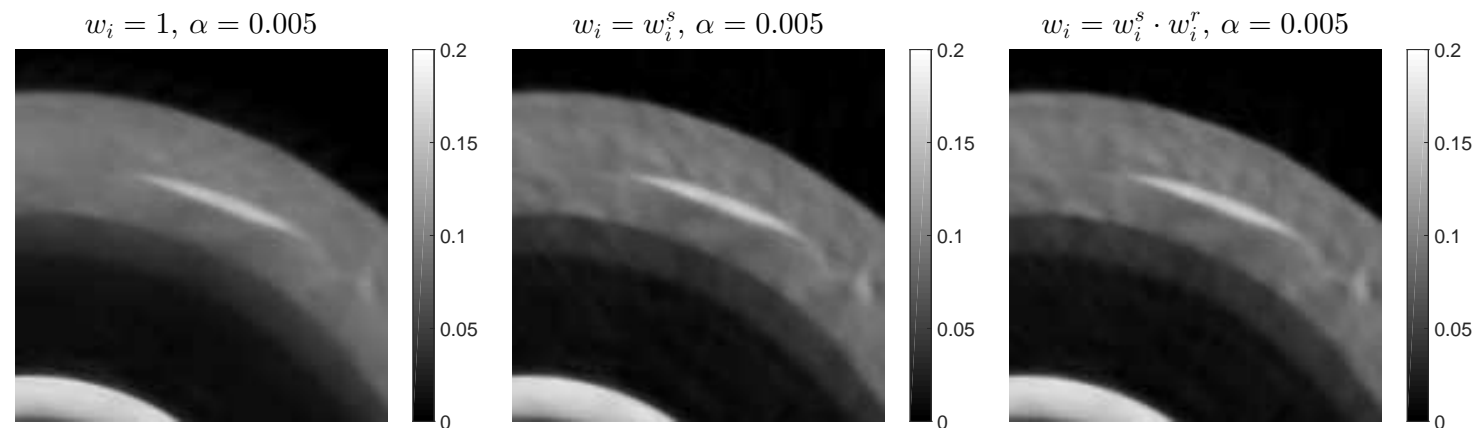

Figure 9. The effect of varying the weights $\boldsymbol{W}$ in our model (8) with 90 projection angles. Left: without the weight ( $\boldsymbol{W}$ equals the identity). Middle: only scale weights $\left(w_{\max }=1\right)$. Right: weight scheme in Section 3.3 and $\left(w_{\max }=5\right)$.

no difference in the quality by adding the ray-density based weights to our method for the off-centered scanning geometry. This is likely because the density of the X-rays is spread evenly over the pipe, in contrast to the centered scanning geometry where the density is much higher in the center region.

\section{Conclusion}

We showed that compared with a centered scanning geometry that only captures the center region, an off-centered scanning geometry is able to capture all material layers and most cracks and defects in an underwater oil pipe, while still using a fan beam that only illuminates half the pipe for each measurement.

In order to reduce the number of measurements in a full rotational scan we proposed a shearlet-based method that is able to maintain better image quality with fewer measurements compared to Kaczmarz's algorithm and the L2-TV method. In addition, we studied parameter choices in our method with the off-centered scanning geometry. We showed that the scale weights on shearlet coefficients have a strong effect on image quality, while the ray-density weights have little influence on the reconstruction, because of the mostly uniform ray density under the off-centered geometry. 


\section{Acknowledgements}

We thank our collaborators Torben Klit Pedersen and Arvid Piehl Lauritsen Böttiger from FORCE Technology, Denmark for their advice and for providing data. We are grateful to Jürgen Frikel and Todd Quinto for their help and valuable insight into microlocal analysis and limited-data problems. We also thank our colleague Martin S. Andersen for his invaluable help with the numerical optimization methods.

This work is a part of the project HD-Tomo funded by Advanced Grant No. 291405 from the European Research Council. The authors also acknowledge the financial support from CINEMA: the allianCe for ImagiNg of Energy MAterials, DSF-grant no. 1305-00032B under the Danish Council for Strategic Research.

\section{References}

[1] E T Quinto. Tomographic reconstructions from incomplete data-numerical inversion of the exterior Radon transform. Inverse Problems, 4(3):867, 1988.

[2] P Kuchment et al. On local tomography. Inverse Problems, 11(3):571, 1995.

[3] Jürgen Frikel and Eric Todd Quinto. Characterization and reduction of artifacts in limited angle tomography. Inverse Problems, 29(12):125007, 2013.

[4] R Felea and E T Quinto. The microlocal properties of the local 3-D SPECT operator. SIAM J. Math. Anal., 43(3):1145, 2011.

[5] E T Quinto. Local algorithms in exterior tomography. Journal of Computational and Applied Mathematics, 199(1):141-148, 2007.

[6] Venkasteswaran P. Krishnan and Eric Todd Quinto. Microlocal analysis in tomography. In Otmar Scherzer, editor, Handbook of Mathematical Methods in Imaging, pages 847-902. Springer-Verlag New York, 2 edition, 2015.

[7] Jürgen Frikel. Sparse regularization in limited angle tomography. Applied and Computational Harmonic Analysis, 34(1):117-141, 2013.

[8] Esther Klann et al. Wavelet methods for a weighted sparsity penalty for region of interest tomography. Inverse Problems, 31(2):025001, 2015.

[9] Bert Vandeghinste et al. Combined shearlet and TV regularization in sparse-view CT reconstruction. In 2nd International Meeting on image formation in X-ray Computed Tomography, Proceedings, page 4, 2012.

[10] E. Garduño and G. T. Herman. Computerized tomography with total variation and with shearlets. Inverse Problems, 33(4):044011, April 2017.

[11] Bert Vandeghinste et al. Iterative CT reconstruction using shearlet-based regularization. IEEE Transactions on Nuclear Science, 60(5):3305-3317, 2013.

[12] T A Bubba et al. The ROI CT problem: a shearlet-based regularization approach. J. Phys.: Conf. Ser., 756(1):012009, 2016.

[13] Gitta Kutyniok and Wang-Q Lim. Compactly supported shearlets are optimally sparse. Journal of Approximation Theory, 163(11):1564-1589, 2011.

[14] Per Christian Hansen and Jakob Sauer Jørgensen. AIR Tools II: algebraic iterative reconstruction methods, improved implementation. Numerical Algorithms, 2017. to appear.

[15] I Daubechies, M Defrise, and C De Mol. An iterative thresholding algorithm for linear inverse problems with a sparsity constraint. Communications on Pure and Applied Mathematics, 57(11):1413-1457, 2004.

[16] Gitta Kutyniok, Jakob Lemvig, and Wang Q. Lim. Compactly supported shearlets. Springer Proceedings in Mathematics, 13:163-186, 2012. 
[17] Demotrio Labate Gitta Kutyniok. Shearlets: Multiscale Analysis for Multivariate Data. Birkhäuser, 1 edition, 2012.

[18] Gitta Kutyniok, Wang-Q Lim, and Rafael Reisenhofer. ShearLab 3D: Faithful digital shearlet transforms based on compactly supported shearlets. ACM Transactions on Mathematical Software, 42(1):1-42, 2016. Available from www.shearlab.org.

[19] Stephen Boyd, Neal Parikh, Eric Chu, Borja Peleato, and Jonathan Eckstein. Distributed optimization and statistical learning via the alternating direction method of multipliers. Foundations and Trends in Machine Learning, 3(1):1-122, 2010.

[20] Åke Björck. Numerical Methods for Least Squares Problems. SIAM, 1996.

[21] NIST X-ray attenuation coefficient tables. Available from www.nist.gov/pml/ $\mathrm{x}$-ray-mass-attenuation-coefficients.

[22] Gabor T. Herman. Fundamentals of Computerized Tomography: Image Reconstruction from Projections. Springer, 2 edition, 2009.

[23] Li Rudin et al. Nonlinear total variation based noise removal algorithms. Physica D, 60(1-4):259268, 1992.

[24] Antonin Chambolle and Thomas Pock. A first-order primal-dual algorithm for convex problems with applications to imaging. Journal of Mathematical Imaging and Vision, 40(1):120-145, 2011. 